\title{
Kinetic Equations for the Collisional Plasma Model
}
W. I. van Rij
H. K. Meier
C. O. Beasley, Jr.
J. E. McCune 


\section{DISCLAIMER}

This report was prepared as an account of work sponsored by an agency of the United States Government. Neither the United States Government nor any agency Thereof, nor any of their employees, makes any warranty, express or implied, or assumes any legal liability or responsibility for the accuracy, completeness, or usefulness of any information, apparatus, product, or process disclosed, or represents that its use would not infringe privately owned rights. Reference herein to any specific commercial product, process, or service by trade name, trademark, manufacturer, or otherwise does not necessarily constitute or imply its endorsement, recommendation, or favoring by the United States Government or any agency thereof. The views and opinions of authors expressed herein do not necessarily state or reflect those of the United States Government or any agency thereof. 


\section{DISCLAIMER}

Portions of this document may be illegible in electronic image products. Images are produced from the best available original document. 


\section{Printed in the United States of America: Available from National Technical Information Service \\ U.S. Department of Commerce \\ 5285 Port Royal Road, Springfield, Virginia 22161 \\ Price: Printed Copy $\$ 4.00$; Microfiche $\$ 2.25$}

This report was prepared as an account of work sponsored by the United States Government. Neither the United States nor the Energy Research and Development Administration/United States Nuclear Regulatory Commission, nor any of their employees, nor any of their contractors, subcontractors, or their employees, makes any warranty, express or implied, or assumes any legal liability or responsibility for the accuracy, completeness or usefulness of any information, apparatus, product or process disclosed, or represents that its use would not infringe privately owned rights. 
Contract No. W-7405-eng-26

THERMONUCLEAR DIVISION

KINETIC EQUATIONS FOR THE COLLISIONAL PLASMA MODEL

W. I. $\operatorname{van}$ Rij, H. K. Meier, C. O. Beasley, Jr.

Oak Ridge National Laboratory

a.n.

J. E. McCune

Massachusetts Institute of Technology

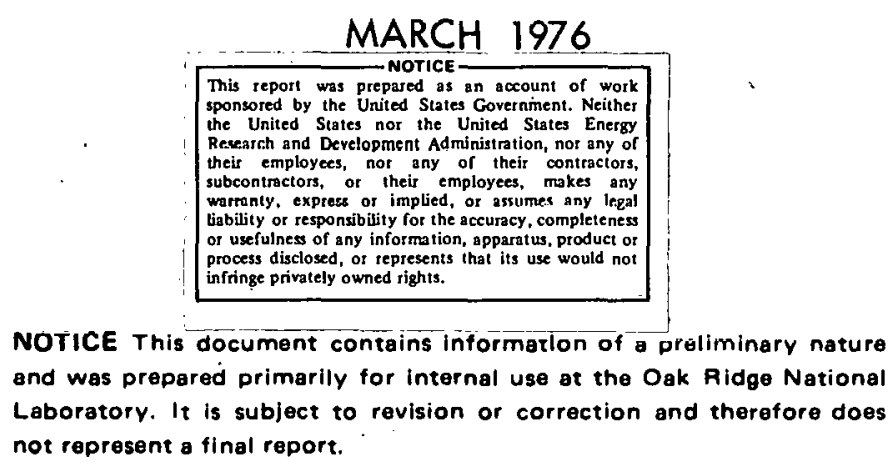

OAK RIDGE NATIONAL I.ABORATORY

Oak Ridge, Tennessee 37830

operated by

UNION CARBIDE CORPORATION

for the

ENERGY RESEARCH AND DEVELOPMENT ADMINISTRATION 
THIS PAGE

\section{WAS INTENTIONALLY LEFT BLANK}


Title

$\underline{\text { Page }}$

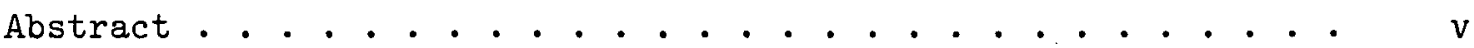

Introduction ...................... . . . 1

Boltzmann Equation in the CPM Representation . . . . . . . . 3

Drift-Kinetic Equation for the CPM . . . . . . . . . . . 7

Drift-Kinetic Equation in the CPM Representation . . . . . . . 13

Drift-Kinetic-Approximation Macroscopic Observables . . . . . 19

Referenges. . . . . . . . . . . . . . . . . . . 22

Appendix - Boltzmann Equation Matrix Elements . . . . . . . 25

Distribution . . . . . . . . . . . . . . . 29 


\section{THIS PAGE \\ WAS INTENTIONALLY \\ LEFT BLANK}




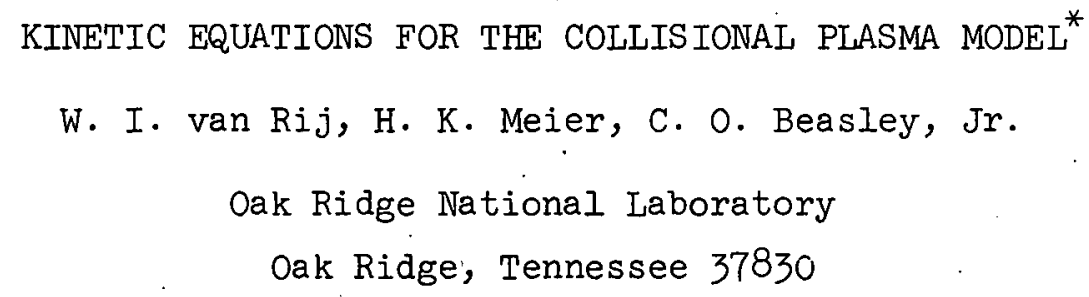

and

J. E. McCune

Massachusetts Institute of Technology

Cambridge, Massachusetts 02139

\begin{abstract}
Using the Collisional Plasma Model (CPM) representation, expressions are derived for the Vlasov operator, both in its general form and in the drift-kinetic approximation following the recursive derivation by Hazeltine. The expressions for the operators give easily calculated couplings between neighboring components of the CPM representation. Expressions for various macroscopic observables in the drift-kinetic approximation are also given.
\end{abstract}

\footnotetext{
${ }^{*}$ Research sponsored by the Energy Research and Development Administration under contract with Union Carbide Corporation.
} 


\section{INTRODUCTION}

The Collisional Plasma Model (CPM) is a velocity-space orthogonalfunction representation for the distribution function of a collisional plasma, which is described in Ref. I (hereafter referred to as I). The CPM assumes that the distribution function for a particular species of particles in a collisional plasma can be represented by the series

$$
f(\vec{x}, \vec{u}, t)=\sum_{\ell=0}^{\infty} \sum_{m=-l}^{\ell} \sum_{n=0}^{\infty} F_{\ell m n}(\vec{x}, t) \psi_{\ell m n}(\sqrt{\rho(\vec{x}, t)} \vec{u})
$$

where

$$
\vec{u}=\vec{v}-v(\vec{x}, t)
$$

$\vec{v}$ being the velocity, $\vec{x}$ the position, and the time. The functions $\psi_{\text {lmn }}$ are defined in $I$; $\rho$ and $\vec{V}$ are essentiaily arbitrary, although in its most useful form, the CPM sets $\rho=M / k T$, with $T$ and $\vec{V}$ equal to the particles' temperature and average velocity. Thus, for predominantly Maxwellian particles, the infinite series (1.1) can be truncated to a practical length.

The choice of the functions $\psi_{\ell m n}$ is determined by several criteria: 1) the functions must provide an accurate representation of the Coulomb collision operator; 2) the representation must be efficient in that physical plasmas be represented by as few terms as possible in order to minimize both computer time and storage requirements; and 3) the collision and Vlasov operators must lead to simple expressions which can be rapidly and easily calculated. These criteria, as they pertain to the Coulomb collision operator, are discussed in $I$. In the present paper, the CPM representation of the Vlasov operator (that is, the 
operator which describes the particle motion in various electric and magnetic fields) is derived for the general kinetic (Boltzmann) equation, and for a drift-kinetic equation of the Hazeltine type, ${ }^{2}$ which is based on the velocity $\vec{u}$ of Eq. (1.2). The various terms of the Vlasov operator give coupling only between neighboring components of the CPM representation, and these couplings are simple in form and easy to calculatc.

Also given in this paper are expressions for macroscopic observables in the drift-kinetic approximation. Presented in another paper 3 is the application of the CPM in the drift-kinetic approximation to the calculation of the neoclassical equilibrium of a toroidal plasma. The success of the calculation indeed bears out the fact that the CPM does satisfy the criteria stated above. 


\section{BOLTZMANN EQUATION IN THE CPM REPRESENTATION}

The general kinetic equation governing the motion of a collisional plasma is the Boltzmann equation

$$
\frac{D f}{D t}=\mathrm{Cf},
$$

where

$$
\begin{gathered}
\frac{\mathrm{D}}{\mathrm{Dt}}=\frac{\partial}{\partial \mathrm{t}}+\overrightarrow{\mathrm{v}} \cdot \vec{\nabla}+(\overrightarrow{\mathrm{a}}+\overrightarrow{\mathrm{v}} \times \vec{\Omega}) \cdot \vec{\nabla}_{\mathrm{v}}, \\
\overrightarrow{\mathrm{a}}=\frac{\mathrm{Ze}}{\mathrm{M}} \overrightarrow{\mathrm{E}}, \quad \vec{\Omega}=\frac{\mathrm{Ze}}{\mathrm{Mc}} \overrightarrow{\mathrm{B}},
\end{gathered}
$$

and $\mathrm{C}$ is the Coulomb collision operator described in I. Two transformations can in general be applied to Eq. (2.1). The first is a transformation to the velocity variable $\vec{u}$ defined by Eq. (1.2). In the second transformation, the action of $\partial / \partial t$ and $\vec{\nabla}$ on the velocity $\vec{u}$ is separated out, these two operators subsequently no longer acting on the $\vec{u}$ dependence of $f$. The reason for the transformation is that, although position is measured in a fixed frame of reference, the velocity frame of reference is often a dynamic one. A third transformation, based on the introduction of the CPM representation (1.1), can also be applied to Eq. (2.1). The artion of $\partial / \partial$ t and $\vec{\nabla}$ on the $\rho$ dependeicie uf the $\psi_{\text {emn }}$ is separated out, these two operators subsequently no longer acting on that $\rho$ dependence. Applying these three transformations to Eq. (2.1), - one obtains

$$
\begin{array}{r}
\frac{D}{D t}=\frac{d}{d t}+\vec{u} \cdot \vec{\nabla}+\vec{A} \cdot \vec{\nabla}_{u}+(G+\vec{H} \cdot \vec{u}) u \frac{\partial}{\partial u} \\
\quad-i\left(\vec{\Omega}^{\prime}+\vec{u} \cdot \stackrel{x}{=} \cdot \vec{L}_{u}-\vec{u} \cdot \underline{W} \cdot \vec{\nabla}_{u},\right.
\end{array}
$$


where

$$
\begin{gathered}
\frac{d}{d t}=\frac{\partial}{\partial t}+\vec{V} \cdot \vec{\nabla}, \\
\vec{A}=\vec{a}+\vec{V} \times \vec{\Omega}-\frac{d \vec{V}}{d t}, \\
G=-\frac{1}{3} \operatorname{div} \vec{V}+\frac{1}{2 p} \frac{d \rho}{d t}, \\
\vec{H}=\frac{1}{2 p} \vec{\nabla} \rho, \\
\vec{\Omega}^{\prime}=\vec{\Omega}+\frac{1}{2} \cdot \operatorname{curl} \vec{V}+\vec{\omega},
\end{gathered}
$$

and

$$
\overrightarrow{\mathrm{L}}_{u}=-i \vec{u} \times \vec{\nabla}_{u}
$$

is the $\vec{u}$-space angular momentum operator. The rectangular Cartesian components of the symmetric traceless tensor $\underset{W}{W}$ are given by

$$
\begin{gathered}
w_{i, j}=\frac{I}{2}\left(w_{i j}+w_{j i}\right)-\frac{1}{3} \delta_{i j} d i v \vec{V}, \\
w=\overrightarrow{\nabla V} .
\end{gathered}
$$

The rectangular Cartesian components of $\vec{\omega}$ in the arbitrary velocity reference frame are given by

$$
\omega_{i}=-\frac{1}{2} \sum_{j k} \epsilon_{i j k} \cdot \ddot{u}_{j} \cdot \frac{d \hat{u}_{k}}{d t},
$$

where $\varepsilon_{i, j k}$ is the permutation symbol and the $\hat{u}_{i}$ dire the velocity-epace unit vectors. The components of the tensor $\stackrel{x}{=}$ are given by

$$
n_{i j}=-\frac{I}{2} \sum_{k \ell} \epsilon_{j k \ell} \hat{u}_{k} \cdot \frac{\partial \hat{u}_{\ell}}{\partial x_{i}} .
$$

It should be noted that $\vec{A}, G, \vec{H}, \vec{\Omega}^{\prime}, \stackrel{n}{=}$, and $\underset{=}{=}$ are functions of $\vec{x}$ and $t$ only. 
Upon substituting from Eq. (1.I) it is possible to obtain the following expansion for $\mathrm{Df} / \mathrm{Dt}, \mathrm{D} / \mathrm{Dt}$ being defined by Eq. (2.2):

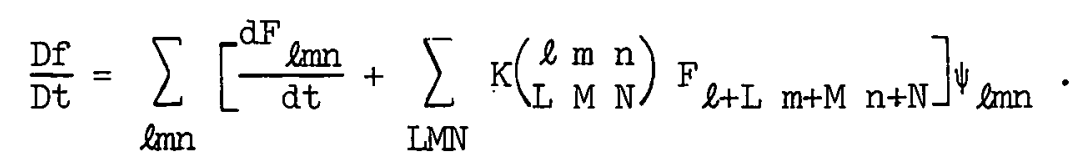

The reality of $f$ and the relation

$$
\psi_{\ell m n}^{*}=(-1)^{m} \psi_{l-m n}
$$

give

$$
\begin{gathered}
\mathrm{F}_{\ell m n}^{*}=(-I)^{\mathrm{m}} \mathrm{F}_{\ell-\mathrm{mn}}, \\
\mathrm{K}\left(\begin{array}{lll}
\ell & \mathrm{m} & \mathrm{n} \\
\mathrm{L} & \mathrm{M} & \mathrm{N}
\end{array}\right)^{*}=(-1)^{\mathrm{M}} \mathrm{K}\left(\begin{array}{lll}
\ell & -\mathrm{m} & \mathrm{n} \\
\mathrm{L} & -\mathrm{M} & \mathrm{n}
\end{array}\right) .
\end{gathered}
$$

The restrictions on $\mathrm{L}, \mathrm{M}$, and $\mathrm{N}$ are

$$
-2 \leq \mathrm{L} \leq 2, \quad-2 \leq \mathrm{M} \leq 2, \quad-2 \leq \mathrm{N} \leq 2,
$$

that is, the Vlasov operator gives coupling only between neighboring components of the CPM representation. Of course, one must also have

$$
\ell+L \geq|m+M|, \quad n+N \geq 0,
$$

but for a given (lmn) there are, according to $\mathrm{Eq} .(2.5)$, at most $125 \mathrm{~K}$ matrix elements. Equation (2.4) can be used to calculate the negative m matrix elements. However, many of the 125 vanish; in fact, there are only 65 non-zero elements for a given $(\ell \mathrm{mn})$. If one chooses $\vec{V}=0$, the number is further reduced to 37 . The non zero elements for a given ( $l m n)$ and $M \geq 0$ are presented in the Appendix, with the notation abbreviated to $K(L M N)$. The $\ell, m$, and $n$ dependence of all the matrix elements is simple in form and easy to calculate. 
THIS PAGE

\section{WAS INTENTIONALLY LEFT BLANK}


3. DRIFT-KINETIC EQUATION FOR THE CPM

For problems characterized by time scales that are much larger than the gyroperiod $\Omega^{-1}$, it is possible to average the Boltzmann equation with respect to the gyromotion to obtain a drift-kinetic equation. Recently, Hazeltine $\hat{\varepsilon}^{\hat{}}$ gave a recursive derivation of such a drift-kinetic equation. Here we give a generalization of that drift-kinetic equation. This generalization is based on the velocity transformation Ea. (1.2), in which some unspecified average or drift velocity $\vec{V}$ is subtracted from the total velocity $\vec{v}$. Thus, the picture is one where the velocity $\vec{u}$ gyrates with frequency $\Omega$ about the $\vec{B}$ field.

Up until this point, the CPM velocity reference frame has been arbitrary, but we now assume that

$$
\hat{\mathrm{u}}_{3}=\hat{\mathrm{n}}=\overrightarrow{\mathrm{B}} / \mathrm{B}
$$

so that $\alpha$ and $\beta$, the spherical polar angles of $\vec{u}$, are the pitch and gyro angles. (The angle $-\beta$ is the counterpart of Hazeltine's 5.) The $\beta$ dependence of the $\psi_{l m n}$ of $\mathrm{Eq}$. (1.I) is $\exp (\operatorname{im} \beta)$ (see I), so that the gyroaveraged distribution function

$$
\bar{\Gamma}=\frac{1}{2 \pi} \int_{0}^{2 \pi} d \beta f
$$

is simply given by the $m=0$ part of Eq. (I.I). Because the $\psi_{\text {lmn }}$ are defined as functions of $u, \alpha$, and $\beta$, it is natural to derive the CPM drift-kinetic equation lu $(u, \dot{x})$ varlables rather than the (energy, magnetic moment) variables used by Hazeltine. 2

our recursive derivation follows closely that given by Hazeltine. 2 However, in treating the Coulomb collisions, we assume that, for species $r$ and $s$, the velocity $\vec{V}_{r}-\vec{V}_{g}$ is negligible in comparison 
to the thermal velocity or parallel to $\vec{B}$. We then obtain the following first-order drift-kinetic distribution function

$$
f=\bar{f}+\tilde{f}_{1}+\tilde{f}_{2},
$$

where $\bar{f}$ is the solution of the drift-kinetic equation (3.6), and

$$
\begin{aligned}
& \tilde{f}_{1}=-\frac{1}{\Omega} \hat{\beta} \cdot {\left[u \sin \alpha\left(\vec{\nabla}+\vec{H} u \frac{\partial}{\partial u}\right)+\vec{A} \hat{s} \cdot \vec{\nabla}_{u}\right.} \\
&-\vec{w}^{\prime} \frac{\partial}{\partial \alpha}+\hat{n} \times \operatorname{curl} \hat{n} u \cos \alpha \frac{\partial}{\partial \alpha} \\
&\left.-2 \vec{d} u \sin \alpha \hat{n} \cdot \vec{\nabla}_{u}\right] \bar{f}, \\
& \tilde{f}_{2}=\frac{1}{4 \Omega} u \sin \alpha\left[(\hat{\beta} \cdot \operatorname{curl} \hat{\beta}-\hat{s} \cdot \operatorname{curl} \hat{s}) \frac{\partial}{\partial \alpha}\right. \\
&\left.+2 \hat{s} \cdot \underline{\underline{W}} \cdot \hat{\beta} \hat{s} \cdot \vec{\nabla}_{u}\right] \bar{f},
\end{aligned}
$$

with

$$
\begin{gathered}
\vec{d}=\hat{n} \cdot \underline{\underline{w}}, \\
\vec{\omega}^{\prime}=\frac{d \hat{n}}{d t}+\hat{n} \cdot \underline{w}, \\
\hat{\beta}=-\sin \beta \hat{u}_{I}+\cos \beta \hat{u}_{2}, \\
\hat{s}=\cos \beta \hat{u}_{I}+\sin \beta \hat{u}_{2} .
\end{gathered}
$$

Three sets of right-handed orthogonal unit vectors are involved here: rectangular vartesian $\left(\hat{u}_{1}, \hat{u}_{2}, \hat{u}_{3}=\hat{n}\right)$, Epherical polar $(\hat{u}, \hat{\alpha}, \hat{\beta})$, and the sel $(\hat{\mathfrak{r}}, \hat{\mathrm{s}}, \hat{\beta})$ with

$$
\begin{aligned}
& \hat{\mathrm{n}}=\cos \alpha \hat{\mathrm{u}}-\sin \alpha \hat{\alpha}, \\
& \hat{\mathrm{s}}=\sin \alpha \hat{\mathrm{u}}+\cos \alpha \hat{\alpha} .
\end{aligned}
$$

It is instructive to consider the time dependence characteristics of $\bar{f}, \tilde{f}_{1}$, and $\tilde{f}_{2}$. For the drift-kinetic derivation, it is assumed that 
the dominant term in the Boltzmann equation is given by the term - $i \vec{\Omega} \cdot \vec{L}_{u}($ see Eq. (2.2)). Thus, if one neglects all other terms of the Boltzmann equation, it follows that the distribution function has a $\beta$ and $t$ dependence which is given by the expansion

$$
f=\sum_{m} f_{m} \exp [i m(\beta+\Omega t)],
$$

so that $m$ exactly identifies the gyroharmonics of $f$. Re-introduction of the neglected terms leads to an expansion of the form

$$
f=\sum_{m M} f_{m M}(t) \exp [i(m \beta+M \Omega t)]
$$

with the diagonal amplitude $f_{\mathrm{mm}}$ dominating. Consequently, m now only approximately identifies the gyroharmonics of $f$. The amplitudes $f_{\mathrm{mM}}$ vary on a time scale much larger than $\Omega^{-1}$; therefore, performing the gyromotion average of the drift-kinetic derivation, one obtains

$$
f\left(\operatorname{arift-kinetic)} \approx \sum_{m} f_{m 0}(t) \exp (i m \beta)\right.
$$

Discarded in this approximation are the possibly large, but rapidly varying $M \neq 0$ terms. In our first-order drift-kinetic approximation we only have $m=0(\bar{f}), m= \pm I\left(\tilde{f}_{1}\right)$, and $m= \pm 2\left(\tilde{f}_{2}\right)$ terms, all varying on the larger time scale.

The first-order drift-kinetic equation is given by

$$
\left[\frac{\partial}{\partial t}+\vec{V}_{\text {tot }} \cdot \vec{\nabla}+\vec{A}_{\text {tot }} \cdot \vec{\nabla}_{u}\right] \bar{f}=\bar{C} \bar{f},
$$

where the collision operator $\bar{C}$ is to be calculated with the $\sqrt{\rho}\left(\vec{V}_{r}-\vec{V}_{s}\right)=0$ or $\hat{n} \cdot\left(\vec{V}_{r}-\vec{V}_{s}\right)=0$ assumption referred to previously. The velocity $\vec{V}_{\text {tot }}$ is given by 


$$
\begin{gathered}
\vec{v}_{\text {tot }}=\vec{v}+\vec{v}_{D}+\left(\hat{n}+\frac{1}{\Omega} \hat{n} \times \vec{w}^{\prime}\right) u \cos \alpha \\
+\frac{1}{2 \Omega}[\hat{n} \times \vec{b}+\hat{n}(\hat{n} \cdot \operatorname{curl} \hat{n})] u^{2} \\
-\frac{1}{2 \Omega}[\hat{n} \times \vec{b}+3 \hat{n}(\hat{u} \cdot \operatorname{curl} \hat{n})-2 \operatorname{curl} \hat{n}] u^{2} \cos ^{2} \alpha,
\end{gathered}
$$

with

$$
\begin{aligned}
& \vec{V}_{D}=\frac{l}{\bar{\Omega}} \vec{A} \times \hat{n}=\vec{V}_{E}-\vec{V}_{\perp}+\frac{1}{\Omega} \hat{n} \times \frac{d \vec{V}}{d t},
\end{aligned}
$$

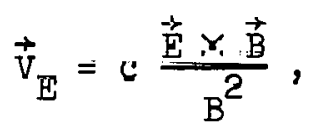

$$
\begin{aligned}
& \vec{V}_{\perp}=\vec{V}-\hat{n}(\hat{n} \cdot \vec{V}) \text {, } \\
& \overrightarrow{\mathrm{b}}=\frac{\mathrm{l}}{\mathrm{B}} \vec{\nabla} \mathrm{B} \text {. }
\end{aligned}
$$

The velocity $\vec{V}_{E}$ is just the electric drift velocity, and $\vec{v}_{\perp}$ is the component of $\vec{V}$ perpendicular to $\vec{B}$. Thus, $\vec{V}_{D}$ is the transverse drift velocity that remains after subtraction of the drift $\vec{V}$ in Eq. (1.1). However, if one chooses $\vec{V}=\vec{V}_{E}$, it does not follow that $\vec{v}_{D}=0$, because of the first-order term in Eq. (3.8).

The acceleration $\vec{A}_{\text {tot }}$ is given by

$$
\begin{aligned}
& \vec{A}_{\text {tot }}=\vec{u}\left\{G+\frac{1}{2} \vec{a} \cdot\left(\hat{n}+\frac{2}{\Omega} \hat{n} \times \vec{\omega}^{\prime}\right)-\frac{1}{2} \operatorname{div} \vec{V}_{D}\right. \\
& +\vec{V}_{D} \cdot\left(\vec{H}+\frac{l}{2} \hat{n} \times \operatorname{curl} \hat{n}\right)-\left[\frac{l}{2} \operatorname{div}\left(\hat{n}+\frac{l}{\Omega} \hat{n} \times \vec{\omega}^{\prime}\right)\right. \\
& \left.-\left(\ddot{n}+\frac{1}{\bar{\Omega}} \hat{n} \times \vec{\omega}^{\prime}\right) \cdot \vec{H}-\frac{?}{\Omega}(\hat{n} \times \vec{d}) \cdot(\hat{n} \times \operatorname{curl} \hat{n})\right] \mathrm{u} \cos \alpha \\
& +\frac{1}{2 \Omega}[\hat{n} \times \vec{b}+\hat{n}(\hat{n} \cdot \operatorname{curl} \hat{n})] \cdot \vec{H} u^{2}-\left[\frac{1}{2} \hat{n} \cdot \operatorname{curl}\left(\frac{1}{\Omega} \hat{n} \times \operatorname{curl} \hat{n}\right)\right. \\
& \left.\left.+\frac{1}{2 \Omega}(\hat{n} \times \vec{b}+3 \hat{n}(\hat{n} \cdot \operatorname{curl} \hat{n})-2 \operatorname{curl} \hat{n}) \cdot \vec{H}\right] u^{2} \cos ^{2} \alpha\right\} \\
& +\hat{n}\left\{\hat{n} \cdot \vec{A}+\left(\vec{w}^{\prime}-2 \vec{d}\right) \cdot \vec{v}_{D}+\left[\frac{1}{2} \operatorname{div} \vec{v}_{D}-\right.\right.
\end{aligned}
$$




$$
\begin{gathered}
\left.-\frac{3}{2} \vec{V}_{D} \cdot(\hat{n} \times \operatorname{curl} \hat{n})-\frac{3}{2} \vec{d} \cdot\left(\hat{n}+\frac{2}{\Omega} \hat{n} \times \vec{w}^{\prime}\right)\right] u \cos \alpha \\
+\left[\frac{1}{2} \operatorname{div}\left(\hat{n}+\frac{1}{\Omega} \hat{n} \times\left(\vec{\omega}^{\prime}-2 \vec{d}\right)\right)-\frac{1}{4 \Omega} \operatorname{Re}\left(P_{2} R_{2}^{*}\right)\right] u^{2} \\
+\left[\operatorname{div}\left(\frac{1}{\Omega} \hat{n} \times \vec{d}\right)-\frac{4}{\Omega}(\hat{n} \times \vec{d}) \cdot(\hat{n} \times \operatorname{curl} \hat{n})\right. \\
\left.+\frac{1}{4 \Omega} \operatorname{Re}\left(P_{2} R_{2}^{*}\right)\right] u^{2} \cos ^{2} \alpha \\
\left.+\frac{1}{2} \hat{n} \cdot \operatorname{curl}\left(\frac{1}{\Omega} \hat{n} \times \operatorname{curl} \hat{n}\right) u^{3} \cos \alpha\right\},
\end{gathered}
$$

where $\mathrm{P}_{2}$ and $\mathrm{R}_{2}$ are defined in the Appendix. This is a rather complicated looking result, but one sees that the velocity dependence is simple, consisting of terms of the type $\dot{u}\left(I, u \cos \alpha, u^{2}, u^{2} \cos ^{2} \alpha\right)$, and $\hat{n}\left(1, u \cos \alpha, u^{2} \cos ^{2} \alpha, u^{3} \cos \alpha\right)$.

The relationship

$$
\operatorname{div} \vec{V}_{\text {tot }}=-\operatorname{div}_{\text {u }} \vec{A}_{\text {tot }}\left(\frac{\partial \rho}{\partial t}=\vec{\nabla}_{-}=0\right)
$$

can readily be derived from Eqs. (3.7) and (3.9). This result is useful for calculating moments of the drift-kinetic equation (3.6). An interesting example is the first moment equation,

$$
\frac{\partial}{\partial t} N(\vec{x}, t)=-\operatorname{div} \vec{\Gamma}(\vec{x}, t)
$$

where

$$
N(\vec{x}, t)=\int a^{3} v \vec{f}
$$

is the number density, and

$$
\vec{\Gamma}(\vec{x}, t)=\int d^{3} v \vec{v}_{\text {tot }} \bar{I}^{\prime}
$$

is a particle flux vector. Furthermore; one can show that

$$
\vec{\Gamma}=\vec{J}+\operatorname{curl}\left(\frac{\hat{n}}{2 \Omega} \int d^{3} u u^{2} \sin ^{2} \alpha \bar{f}\right)
$$


12

where

$$
\vec{J}(\vec{x}, t)=\int d^{3} v \vec{v}\left(\bar{f}+\tilde{f}_{1}+\tilde{f}_{2}\right)
$$

is the particle current density. It follows from Eq. (3.1I) that

Eq. (3.10) can be written in the more familiar form

$$
\frac{\partial}{\partial t} N(\vec{x}, t)=-\operatorname{div} \vec{J}(\vec{x}, t)
$$

and it should be emphasized that $\vec{J}$ is the physical quantity, not $\vec{F}$.

The differing of $\vec{\Gamma}$ and $\vec{J}$ reflects the fact that the arift-kinetic

approximation gives a guiding-center solution (Eq. (3.5)), and not an

exact solution (Eq. (3.4)) . 


\section{DRIFT-KINETIC EQUATION IN THE CPM REPRESENTATION}

Using Eqs. (3.7) and (3.9), the drift-kinetic equation (3.6) can be written in the form

$$
\frac{\partial \bar{f}}{\partial t}=\bar{C} \bar{f}-\sum_{j=1}^{13} K_{j}\left(\sqrt{\rho u}, \frac{1}{\sqrt{\rho}} \vec{\nabla}_{u}\right) s_{j}(\vec{x}, \vec{\nabla}, t) \bar{f} .
$$

The $\mathrm{K}_{j}$ are dimensionless velocity-space operators which are defined as follows:

$$
\begin{aligned}
& \mathrm{K}_{1}=1 \text {, } \\
& \mathrm{K}_{2}=\sqrt{\rho} u \cos \alpha \text {, } \\
& \mathrm{K}_{3}=\rho \mathrm{u}^{2} \\
& K_{4}=\rho u^{2} \cos ^{2} \alpha \text {, } \\
& \mathrm{K}_{5}=\mathrm{u} \frac{\partial}{\partial u} \text {, } \\
& K_{6}=\sqrt{\rho} u^{2} \cos \alpha \frac{\partial}{\partial u} \text {, } \\
& \kappa_{7}=\rho u^{3} \frac{\partial}{\partial u} \text {, } \\
& K_{8}=\rho u^{3} \cos ^{2} \alpha \frac{\partial}{\partial u},: \\
& K_{9}=\frac{1}{\sqrt{\rho}} \hat{n} \cdot \vec{\nabla}_{u}, \\
& \mathrm{~K}_{10}=u \cos \alpha \hat{\mathrm{n}} \cdot \vec{\nabla}_{\mathrm{u}} \text {, } \\
& \mathrm{K}_{11}=\sqrt{\rho} \mathrm{u}^{2} \hat{\mathrm{n}} \cdot \vec{\nabla}_{\mathrm{u}} \text {, } \\
& K_{12}=\sqrt{\rho} u^{2} \cos ^{2} \alpha \hat{n} \cdot \vec{\nabla}_{u} \text {, } \\
& K_{13}=\rho u^{3} \cos \alpha \hat{n} \cdot \vec{\nabla}_{u} \text {. }
\end{aligned}
$$

The definitions of the spatial operators $\mathrm{s}_{j}$ follow directly from a comparison of Eqs. (4.1) and. (4.2) with Eqs. (3.6), (3.7), and (3.9). The 
advantage of the factorization in Eq. (4.1) lies in the fact that only the $\mathrm{S}_{j}$ depend on the nature of the physical problem considered, so that a large class of problems could be studied by the same general computer code.

The gyroaveraged distribution function has a CPM representation given by Eqs. (1.1) and (3.1):

where

$$
\bar{f}(\vec{x}, \vec{u}, t)=\sum_{l=0}^{\infty} \sum_{n=0}^{\infty} F_{l n}(\vec{x}, t) \psi_{l n}(\sqrt{\rho(\vec{x}, t)} \vec{u}) .,
$$

$$
F_{l n}=F_{l O n}, \quad \psi_{l n} \equiv \psi_{l O n} .
$$

As in Eq. (2.3), one can make expansions

$$
K_{j} \bar{f}=\sum_{l n}\left[\sum_{L N} K_{j}\left(\begin{array}{ll}
l & n \\
L & N
\end{array}\right) F_{l+L} n+N\right] \psi_{l n} .
$$

The $K_{j}$ matrices are sparse, with

$$
-3 \leq \mathrm{L} \leq 3, \quad-3 \leq N \leq 3,
$$

subject to the constraints

$$
l+\mathrm{L} \geq 0, \quad \mathrm{n}+\mathrm{N} \geq 0
$$

Comparing Eq. (4.4) with Eq. (2.5), one sees that a wider $L, N$ range is spanned by the drift-kinetic matrix elements. This is because, in the drift-kinetic approximation, some of the $M \neq 0$ coupling of Eq. (2.3) is converted to $\mathrm{L}, \mathrm{N}$ coupling. The $\mathrm{K}_{j}$ matrix elements are pure numbers, and the non-zero elements for a given $(l n)$ are presented below with the notation abbreviated to $\mathrm{K}_{j}(\mathrm{LN})$. All 98 of them are simple in form and easy to calculate. If one chooses $\vec{V}=0$, the spatial operator $\mathrm{S}_{12}$ vanishes, effectively reducing the number of $\mathrm{K}_{j}$ matrix elements to 82 
15

and the $L$ coupling restriction to $-2 \leq L \leq 2$.

$$
\begin{gathered}
\mathrm{K}_{\mathrm{I}}(\mathrm{ON})=\delta_{\mathrm{NO}} . \\
\mathrm{K}_{2}(-\mathrm{IN})=\left(\delta_{\mathrm{NO}}-\delta_{\mathrm{NI}}\right) \mathrm{D}_{l}, \\
\mathrm{~K}_{2}(\mathrm{IN})=-\delta_{-N I} \mathrm{G}_{l n}-\delta_{\mathrm{NO}} \mathrm{H}_{l n},
\end{gathered}
$$

where

$$
\begin{aligned}
& \mathrm{D}_{\ell}=\frac{\ell}{\sqrt{(2 \ell-1)(2 \ell+1)}} \\
& \mathrm{G}_{\ell n}=\mathrm{n}_{\ell+1}, \mathrm{H}_{\ell n}=\left(l+n+\frac{3}{2}\right) \mathrm{D}_{\ell+1} \text {. } \\
& K_{3}(\text { ON })=-\delta_{-N 1}+\delta_{N O}\left(l+2 n+\frac{3}{2}\right)-\delta_{N 1}\left(l+n+\frac{3}{2}\right) . \\
& \mathrm{K}_{4}(-2 \mathrm{~N})=\left(\delta_{\mathrm{NO}}-2 \delta_{\mathrm{N} 1}+\delta_{\mathrm{N} 2}\right) \mathrm{J}_{\ell}, \\
& \mathrm{K}_{4}(\mathrm{ON})=\mathrm{K}_{3}(\mathrm{ON}) \mathrm{I}_{\ell} \\
& \mathrm{K}_{4}(2 \mathrm{~N})=\delta_{-\mathrm{N} 2} G_{\ln } \mathrm{G}_{\ell+1 \mathrm{n}-1}-2 \delta_{-\mathrm{N} 1} \mathrm{G}_{\ell+\mathrm{ln}} \mathrm{H}_{\ell \ln } \\
& +\delta_{\mathrm{NO}} \mathrm{H}_{\ell n} \mathrm{H}_{\ell+\ln } \text {, }
\end{aligned}
$$

where

$$
\begin{gathered}
J_{l}=\mathrm{D}_{l-1} \mathrm{D}_{l}, \quad \mathrm{I}_{\ell}=\mathrm{D}_{\ell}^{2}+\mathrm{D}_{l+1}^{2} \cdot \\
\mathrm{K}_{5}(\mathrm{ON})=\delta_{-\mathrm{N} 1} \mathrm{n}-\frac{3}{2} \delta_{\mathrm{NO}}-\delta_{\mathrm{N} 1}\left(l+\mathrm{n}+\frac{3}{2}\right) \cdot \\
\mathrm{K}_{6}(-\mathrm{IN})=\left[\delta_{-\mathrm{N} 1} \mathrm{n}-\delta_{\mathrm{NO}}\left(\mathrm{n}+\frac{5}{2}\right)-\delta_{\mathrm{N} 1}(l+\mathrm{n}-1)\right. \\
\left.\quad+\delta_{\mathrm{N} 2}\left(l+\mathrm{n}+\frac{3}{2}\right)\right] \mathrm{D}_{l}, \\
\mathrm{~K}_{6}(\mathrm{IN})=-\left[\delta_{-\mathrm{N} 2}(\mathrm{n}-1)-\delta_{-\mathrm{N} I}(l+\mathrm{n}+3)\right] \mathrm{G}_{l n} \\
+\left[\delta_{\mathrm{NO}}\left(\mathrm{n}-\frac{3}{2}\right)-\delta_{\mathrm{N} 1}\left(l+\mathrm{n}+\frac{5}{2}\right)\right] \mathrm{H}_{\ell n} \cdot
\end{gathered}
$$


16

$$
\begin{aligned}
& K_{7}(O N)=-\delta_{-N 2} n(n-1)+\delta_{-N 1} n(l+2 n+3) \\
& -\delta_{\mathrm{NO}} \frac{5}{2}\left(l+2 \mathrm{l}+\frac{3}{2}\right)-\delta_{\mathrm{NI}}(l+2 \mathrm{n})\left(l+\mathrm{n}+\frac{3}{2}\right) \\
& +\delta_{\mathrm{N} 2}\left(l+\mathrm{n}+\frac{3}{2}\right)\left(l+\mathrm{n}+\frac{5}{2}\right) . \\
& K_{8}(-2 N)=\left[\delta_{-N I} n-\delta_{N O}\left(2 n+\frac{7}{2}\right)-\delta_{N 1}\left(l-\frac{11}{2}\right)\right. \\
& \left.+\delta_{N 2}\left(2 l+\cos ^{\prime}-\frac{1}{2}\right)-\sigma_{\mathrm{N} 3}\left(l+\mathrm{n}+\frac{3}{2}\right)\right] J_{l}, \\
& \mathrm{~K}_{8}(\mathrm{ON})=\mathrm{K}_{7}\left(\mathrm{ON}^{\circ}\right) \mathrm{I}_{\ell},
\end{aligned}
$$

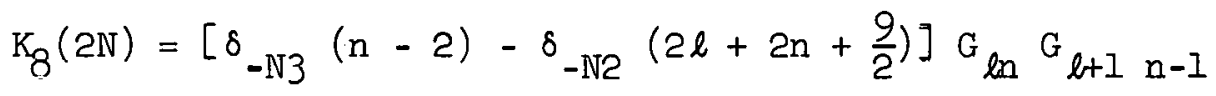

$$
\begin{aligned}
& +\delta_{-\mathrm{N} I}\left(l+\frac{13}{2}\right) \mathrm{G}_{\ell+\ln } \mathrm{H}_{\ell n}+\left[\delta_{\mathrm{NO}}\left(2 \mathrm{n}-\frac{3}{2}\right)-\delta_{\mathrm{NI}}\left(l+\mathrm{n}+\frac{7}{2}\right)\right] \mathrm{H}_{l n} \mathrm{H}_{\ell+\ln } \text {. } \\
& K_{9}(-1 N)=-\left(\delta_{N O}+\delta_{N 1}\right) D_{\ell}, \\
& \mathrm{K}_{9}(\mathrm{IN})-\delta_{-\mathrm{NI}} \mathrm{G}_{\ln }+\delta_{\mathrm{NO}} \mathrm{H}_{\ln \mathrm{i}}: \\
& K_{10}(-2 N)=-\left(\delta_{N O}-\delta_{N^{\prime}}\right) \cdot \ell_{1}, \\
& K_{I O}(\mathrm{ON})=\delta_{-N 1} \mathrm{n} I_{\ell}-\frac{1}{2} \delta_{N O}-\delta_{N 1}\left(l+n+\frac{3}{2}\right) I_{\ell}, \\
& \mathrm{K}_{10}(2 \mathrm{~N})=-\delta_{\mathrm{N} 2} \mathrm{G}_{\ell \mathrm{ln}} \mathrm{G}_{\ell+1 \mathrm{n}-1}+\delta_{\mathrm{NO}} \mathrm{H}_{\ell n} \mathrm{II}_{\ell+1 \mathrm{n}} . \\
& K_{11}(-1 \mathrm{~N})=\left[\left(\delta_{-\mathrm{N} I}-\delta_{\mathrm{N} I}\right) \mathrm{n}-\left(\delta_{\mathrm{NO}}-\delta_{\mathrm{N} 2}\right)\left(l+\mathrm{n}+\frac{3}{2}\right)\right] \mathrm{D}_{l}, \\
& K_{I I}(1 N)=-\left[\delta_{-N 2}(n-1)-\delta_{-N 1}(n+1)\right] G_{l n} \\
& +\left[\delta_{\mathrm{NO}}\left(l+\mathrm{n}+\frac{1}{2}\right)-\delta_{\mathrm{N} 1}\left(l+\mathrm{n}+\frac{5}{2}\right)\right] \mathrm{H}_{\ln } .
\end{aligned}
$$


17

$$
\begin{aligned}
& K_{12}(-3 N)=-\left[\delta_{N O}-\delta_{N 1}-\delta_{N 2}+\delta_{N 3}\right] D_{\ell} J_{\ell-I}, \\
& K_{12}(-I N)=\delta_{-N 1}\left[I_{\ell}+D_{\ell-I}^{2}\right] G_{\ell-I n} \\
& -\delta_{\mathrm{NO}}\left[\mathrm{D}_{\ell+1} \mathrm{H}_{\ell n}+\frac{1}{2}+(\mathrm{n}+1) \mathrm{I}_{\ell-1}\right] \mathrm{D}_{\ell} \\
& -\delta_{N 1}\left[D_{\ell+I} G_{l n}-\frac{I}{2}+\left(l+n+\frac{1}{2}\right) I_{\ell-I}\right] D_{\ell} \\
& +\delta_{\mathrm{N} 2}\left[\mathrm{I}_{\ell}+\mathrm{D}_{\ell-1}^{2}\right] \mathrm{H}_{\ell-1 \mathrm{n}+1} \text {, } \\
& K_{12}(1 N):=-\delta_{-N 2}(n-I)\left[I_{\ell}+D_{\ell+2}^{2}\right] G_{l n}
\end{aligned}
$$

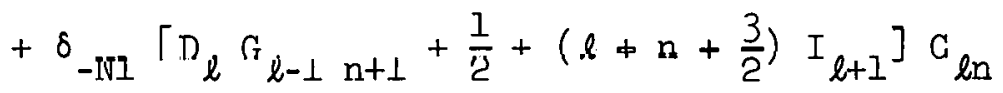

$$
\begin{aligned}
& +\delta_{\text {NO }}\left[\mathrm{D}_{\ell}{ }_{\ell-\mathrm{In}}-\frac{1}{2}+\mathrm{n} \mathrm{I}_{\ell+1}\right] \mathrm{H}_{\ell n} \\
& -\delta_{\mathrm{N} I}\left(l+\mathrm{n}+\frac{3}{2}\right)\left[\mathrm{I}_{\ell}+\mathrm{D}_{\ell+2}^{2}\right] \mathrm{H}_{\ell n+1} \text {, }
\end{aligned}
$$

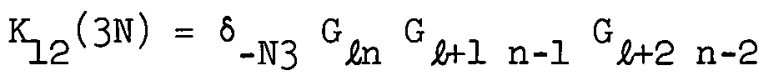

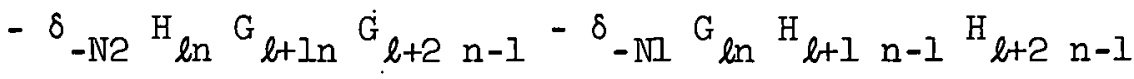

$$
\begin{aligned}
& +\delta_{\mathrm{NO}}{ }^{\mathrm{H}} \ln { }^{\mathrm{H}} \ell+\ln { }^{\mathrm{H}} \ell+2 \mathrm{n} \\
& K_{13}(-2 N)=\left[\delta_{-N 1} n-\delta_{N O}\left(l+2 n+\frac{3}{2}\right)+\delta_{N 1}\left(l+\frac{3}{2}\right)\right. \\
& \left.+\delta_{\mathrm{N} 2}\left(l+2 \mathrm{n}+\frac{3}{2}\right)-\delta_{\mathrm{N} 3}\left(l+\mathrm{n}+\frac{3}{2}\right)\right] J_{\ell}, \\
& K_{13}(\mathrm{ON})=-\delta_{-N 2} \mathrm{n}(\mathrm{n}-1) \mathrm{I}_{\ell}+\delta_{-\mathrm{N} 1} \mathrm{n}\left[\frac{1}{2}+\left(l+2 \mathrm{n}+\frac{3}{2}\right) \mathrm{I}_{\ell}\right] \\
& -\delta_{\mathrm{NO}}\left(l+2 n+\frac{3}{2}\right)\left(\frac{1}{2}+I_{\ell}\right)+\delta_{\mathrm{N} 1}\left(l+n+\frac{3}{2}\right)\left[\frac{1}{2}-\left(l+2 n+\frac{3}{2}\right) I_{\ell}\right] \\
& +\delta_{\mathrm{N} 2}\left(l+\mathrm{n}+\frac{3}{2}\right)\left(l+\mathrm{n}+\frac{5}{2}\right) I_{\ell},
\end{aligned}
$$


18

$$
\begin{gathered}
\left.\mathrm{K}_{13}(2 \mathrm{~N})=\delta_{-\mathrm{N} 3}(\mathrm{n}-2)-\delta_{-\mathrm{N} 2}\left(l+2 \mathrm{n}+\frac{3}{2}\right)\right] \mathrm{G}_{l n} \mathrm{G}_{\ell+1 \mathrm{n}-1} \\
-\delta_{-\mathrm{N} 1}\left(l-\frac{1}{2}\right) \mathrm{G}_{\ell n} \mathrm{H}_{\ell+1 \mathrm{n}-1}+\left[\delta_{\mathrm{NO}}\left(l+2 \mathrm{n}+\frac{3}{2}\right)\right. \\
\left.-\delta_{\mathrm{N} 1}\left(l+\mathrm{n}+\frac{7}{2}\right)\right] \mathrm{H}_{\ln } \mathrm{H}_{\ell+\ln } .
\end{gathered}
$$




\section{DRIFT-KINETIC-APPROXIMATION MACROSCOPIC OBSERVABLES}

Expressions for macroscopic observables given in I are in terms of the expansion amplitudes of the exact distribution function. It is possible to determine the $m= \pm 1, \pm 2$ amplitudes from Eqs. (3.2) and (3.3), and to use these and the $m=0$ amplitudes of Eq. (4.3) in the general equations of I to calculate the drift-kinetic macroscopic observables. However, by substituting directly from Eqs. (3.2) and (3.3) in the appropriate integrals, integration by parts can be used to make simplifications before introducing the expansion Eq. (4.3) for $\bar{f}$. This is the approach that. we have followed in derlvluy the expressions that are given below.

All the observables of interest involve integrals of the type

$$
\begin{aligned}
& I_{s}(l, q)=\int a^{3} u u^{b+2 q} P_{l}(\cos \alpha) \bar{f}_{s} \\
& =\sqrt{\frac{\pi}{\ell+\frac{1}{2}}}\left(\frac{1}{\sqrt{\rho_{s}}}\right)^{b+2 q+3} 2^{l+q+1} \Gamma\left(l+q+\frac{3}{2}\right) \\
& \times \sum_{n=0}^{\infty} F_{l n}^{(s)}(-1)^{n}\left(\begin{array}{c}
b+n+\frac{1}{2} \\
n
\end{array} 2_{2} \Gamma_{1}\left(-n,-q ; l+\frac{3}{2} ; 2\right),\right.
\end{aligned}
$$

where s denotes a particular species, $\mathrm{P}_{\ell}$ is a Legendre polynomial, $\left(\begin{array}{l}a \\ b\end{array}\right)$ is a binomial coefficient, and $2^{F_{1}}$ is the Gauss hypergeometric function.

\subsection{Number Density}

The number density for a species $s$ is. given by

$$
N_{s}=\int d^{3} \mathrm{v}_{s}=I_{s}(0,0)
$$

The plasma number density and average mass are given by 


$$
\begin{gathered}
20 \\
N=\sum_{s} N_{s}, \\
M=\sum_{s} M_{s} N_{s} / N,
\end{gathered}
$$

\subsection{Particle Current Density}

The particle current density for a species $s$ is given by

$$
\vec{J}=d^{3} v \vec{v} f_{s}=N_{s} \vec{v}_{s}+\vec{j}_{s},
$$

where

$$
\begin{gathered}
\vec{j}_{s}=\int d^{3} u \vec{u} f_{s} \\
=N_{s} \vec{V}_{D_{s}}+\left(\hat{n}+\frac{1}{\Omega_{s}} \hat{n} \times \vec{w}_{s}^{\prime}\right) I_{s}(1,0) \\
+\frac{1}{M_{s} \Omega_{s}}\left[\hat{n} \times\left(\vec{\nabla}-5 \vec{H}_{s}\right) w_{\perp s}+(\hat{n} \times \operatorname{curl} \hat{n}) \times \hat{n} D_{s}\right], \\
D_{s}=M_{s} I_{s}(2,0) .
\end{gathered}
$$

The transverse energy density $w_{\perp s}$ is defined in sect. 5.3. The species average velocity is given by

$$
\vec{U}_{S}=\vec{J}_{s} / N_{s}=\vec{V}_{s}+\vec{j}_{s} / N_{S}
$$

and the plasma average velocity is given by

$$
\vec{U}=\sum_{S} M_{S} N_{S} \ddot{U}_{S} / M N
$$

\section{$5 \cdot 3$ Energy Density}

The energy density for a species $s$ is given by

$$
\begin{gathered}
w_{s}=\frac{I}{2} M_{s} \int d^{3} v v^{2} f_{s} \\
=-\frac{1}{2} M_{s} N_{s}\left(v_{s}^{2}-2 \vec{V}_{s} \cdot \vec{U}_{s}\right)+w_{s},
\end{gathered}
$$

where

$$
w_{s}=\frac{1}{2} M_{s} \int d^{3} u u^{2} \cdot \bar{f}_{s}=\frac{1}{2} M_{s} I_{s}(0,1)
$$


The tranverse energy density $w_{\perp s}$ is given by

$$
\begin{gathered}
w_{\perp s}=\frac{1}{2} M_{s} \int d^{3} u u^{2} \sin ^{2} \alpha \bar{f}_{s} \\
=\frac{2}{3} w_{s}-\frac{1}{3} D_{s} .
\end{gathered}
$$

The plasma energy density is given by

$$
\mathrm{W}=\sum_{\mathrm{s}} \mathrm{W}_{\mathrm{S}} \cdot
$$

\subsection{Scaling Pressure and Temperature}

The partial scalar pressure for a species $\mathrm{s}$ is given by

$$
\begin{aligned}
& P_{s}=\frac{1}{3} M_{s} \int d^{3} v(\vec{v}-2 \vec{U})^{2} f_{s} \\
= & \frac{2}{3} W_{s}+\frac{1}{3} M_{s} N_{s}\left(J^{2}-\cdot \vec{U} \cdot \vec{U}_{s}\right) .
\end{aligned}
$$

The plasma scalar pressure is given by

$$
\mathrm{P}=\sum_{\mathrm{s}} \mathrm{P}_{\mathrm{S}}=\frac{2}{3} \mathrm{~W}-\frac{1}{3} \mathrm{MNU}^{2} .
$$

Species and plasma temperatures are given by

$$
\mathrm{kT}_{\mathrm{s}}=\mathrm{P}_{\mathrm{s}} / \mathrm{N}_{\mathrm{s}}, \quad \mathrm{kT}=\mathrm{P} / \mathrm{N} .
$$

\subsection{Tensor Pressure}

The plasma tensor pressure is given by

$$
\begin{aligned}
& \underline{\underline{P}}=\sum_{s} M_{s} \int d^{3} v(\vec{v}-\vec{U})(\vec{v}-\vec{U}) f_{s}-\underline{I} P \\
& =\sum_{s} \underline{p}_{s}-\frac{2}{3} \equiv w_{s}-M_{s} N_{s}\left[\vec{v}_{s} \vec{v}_{s}+\vec{U} \vec{U}-\vec{V}_{s} \vec{U}_{s}\right. \\
& \left.\left.-\vec{U}_{s} \vec{V}_{s}-\frac{1}{3} \equiv\left(v_{s}^{2}+u^{2}-2 \vec{v}_{s} \cdot \vec{U}_{s}\right)\right]\right\} \text {, }
\end{aligned}
$$

where

$$
\stackrel{p}{=}_{s}=M_{s} \int d^{3} u \vec{u} \vec{u} f_{s} .
$$


The components of the tensor $\underline{p}_{s}$ are given by

$$
\begin{gathered}
p_{11}^{(s)}-p_{22}^{(s)}=\frac{1}{\Omega_{s}}\left[\operatorname{Re}\left(R_{2}\right) g_{s}-\operatorname{Im}\left(P_{2}\right) w_{L s}\right], \\
p_{11}^{(s)}+p_{22}^{(s)}=2 w_{s}-p_{33}^{(s)}=2 w_{L s}, \\
p_{12}^{(s)}=\frac{1}{2 \Omega_{s}}\left[\operatorname{Im}\left(R_{2}\right) g_{s}+\operatorname{Re}\left(p_{2}\right) w_{\perp s}\right], \\
\hat{u}_{1} p_{13}^{(s)}+\hat{u}_{2} p_{23}^{(s)}=M_{s} \vec{v}_{D_{s}} I_{s}(1,0)+\frac{1}{\Omega_{s}}\left[\hat{n} \times \vec{\omega}_{s}^{\prime} D_{s}\right. \\
\left.+\hat{n} \times\left(\vec{\nabla}-6 \vec{H}_{s}\right) g_{s}+(\hat{n} \times \operatorname{cirl} \hat{i}) \times \hat{n} h_{s}\right],
\end{gathered}
$$

where $P_{2}$ and $R_{2}$ are defined in the Appendix and

$$
\begin{aligned}
& g_{s}=\frac{1}{5} M_{s}\left[I_{s}(1,1)-I_{s}(3,0)\right], \\
& h_{s}=\frac{1}{5} M_{s}\left[I_{s}(1,1)+4 I_{s}(3,0)\right] .
\end{aligned}
$$

\subsection{Heat Flux Vector}

The plasma heat flux vector is given by

$$
\begin{aligned}
\vec{Q}= & \frac{1}{2} \sum_{s} M_{s} \int d^{3} v(\vec{v}-\vec{U})^{P}(\vec{v}-\vec{U}) I_{s} \\
=-(\underline{p} & +\underline{=} W) \cdot \vec{U}+\sum_{s}\left[\left(\underline{\underline{p}}_{s}+\underline{\underline{1}} w_{s}\right) \cdot \vec{V}_{s}\right. \\
& \left.+\vec{q}_{s}+\frac{1}{?} M_{s} N_{s} v_{s}^{2}\left(\vec{U}_{s}-\vec{v}_{s}\right)\right],
\end{aligned}
$$

where

$$
\begin{gathered}
\vec{q}_{s}=\frac{1}{2} M_{s} \int d^{3} u u^{2} \vec{u} f_{s} \\
=\frac{1}{2} M_{s}\left(\hat{n}+\frac{1}{\Omega_{s}} \hat{n} \times \vec{w}_{s}^{\prime}\right) I_{s}(1, I)+\left(w_{s}+w_{L s}\right) \vec{v}_{D_{s}} \\
+\frac{2}{\Omega_{s}} \hat{n} \times \vec{d}_{s} g_{s}+\frac{M_{s}}{\hat{\Omega}_{s}}\left[\frac{1}{6} \hat{n} \times\left(\vec{\nabla}-7 \vec{H}_{s}\right)\right. \\
\left.\times\left[I_{S}(0,2)-I_{s}(2,1)\right]+\frac{1}{2}(\hat{n} \times \operatorname{curl} \hat{n}) \times \hat{n} I_{s}(2,1)\right] .
\end{gathered}
$$


REFERENCES

1. H. K. Meier, W. I. van Rij, C. O. Beasley, Jr., and J. E. McCune, The Collisional Plasma Model: A Velocity-Space Orthogonal-Function Representation for the Distribution Function of a Collisional Plasma, ORNL/TM-5314 (March 1976).

2. R. D. Hazeltine, Plasma Phys. 15, 77 (1973).

3. C. O. Beasley, Jr., J. E. McCune, H. K. Meier, and W. I. van Rij, Numerical Study of Drift-Kinetic Evolution of Collisional Plasmas in Tori, ORNL/TM-5317 (March 1976). 
THIS PAGE

\section{WAS INTENTIONALLY \\ LEFT BLANK}




\section{APPENDIX - BOLTZMANN EQUATION MATRIX ELEMENTS}

Below are given the non-zero matrix elements defined by Eq. (2.3). Some of them involve the previously undefined quantities

$$
\begin{aligned}
& P_{0}=w_{33}-\frac{1}{2}\left(w_{11}+w_{22}\right), \\
& P_{1}=w_{13}+w_{31}+i\left(w_{23}+w_{32}\right), \\
& P_{2}=w_{11}-w_{22}+i\left(w_{12}+w_{21}\right), \\
& R_{0}=s_{33}-\frac{1}{2}\left(s_{11}+s_{22}\right), \\
& R_{1}=s_{13}+s_{31}+i\left(s_{23}+s_{32}\right), \\
& R_{2}=s_{11}-s_{22}+i\left(s_{12}+s_{21}\right),
\end{aligned}
$$

where

$$
\begin{aligned}
& s_{i j}=\hat{u}_{i} \cdot \operatorname{curl} \hat{u}_{j} \cdot \\
& \mathrm{K}(\mathrm{OON})=-\delta_{\mathrm{NO}}\left(\frac{3}{2} \mathrm{G}+\mathrm{im} \Omega_{3}^{\prime}\right)-\left[\left(l+\mathrm{n}+\frac{3}{2}\right) \delta_{\mathrm{NI}}\right. \\
& \left.-n \delta_{-N 1}\right]\left[G-\frac{2 l(l+1)-6 m^{2}}{3(2 l-1)(2 l+3)} P_{0}\right] \text {, } \\
& K(O I N)=\sqrt{(\ell-m)(\ell+m+1)}\left\{\frac{1}{2} \delta_{N O}\left(\Omega_{2}^{\prime}-i \Omega_{1}^{\prime}\right)\right. \\
& \left.-\frac{\mathrm{m}+1 / 2}{(2 l-1)(2 \ell+3)}\left[\left(l+\mathrm{n}+\frac{3}{2}\right) \delta_{\mathrm{N} 1}-\mathrm{n} \delta_{-\mathrm{NI}}\right] \mathrm{P}_{1}\right\} \text {, } \\
& \mathrm{K}(02 \mathrm{~N})=-\frac{\sqrt{(\ell-\mathrm{m})(\ell-\mathrm{m}-1)(\ell+\mathrm{m}+1)(\ell+\mathrm{m}+2)}}{2(2 \ell-1)(2 \ell+3)} \times\left[\left(\ell+\mathrm{n}+\frac{3}{2}\right) \delta_{\mathrm{NI}}-\mathrm{n} \delta_{-\mathrm{NI}}\right] \mathrm{P}_{2} . \\
& \mathrm{K}(20 \mathrm{~N})=-\frac{1}{2 \ell+3} \sqrt{\frac{(\ell+2-\mathrm{m})(\ell+1-\mathrm{m})(\ell+2+\mathrm{m})(\ell+1+m)}{(2 \ell+1)(2 \ell+5)}} \\
& \times\left[\left(l+\mathrm{n}+\frac{3}{2}\right)\left(l+\mathrm{n}+\frac{5}{2}\right) \delta_{\mathrm{NO}}-\mathrm{n}(\mathrm{n}-1) \delta_{-\mathrm{N} 2}\right] \mathrm{P}_{\mathrm{O}},
\end{aligned}
$$


26

$$
\begin{aligned}
& K(21 N)=\frac{1}{2(2 b+3)} \sqrt{\frac{(l+1-m)(l+1+m)(t+2+m)(l+3+m)}{(2 l+1)(2 l+5)}} . \\
& \times\left[\left(l+n+\frac{3}{2}\right)\left(l+n+\frac{5}{2}\right) \delta_{N O}-n(n-1) \delta_{-N 2}\right] P_{1}, \\
& K(22 N)=-\frac{1}{4(2 b+3)} \sqrt{\frac{(b+1+m)(b+2+m)(b+3+m)(b+4+m)}{(2 b+1)(2 b+5)}} \\
& \times\left[\left(l+n+\frac{3}{2}\right)\left(l+n+\frac{5}{2}\right) \delta_{N O}-n(n-1) \delta_{-N 2}\right] P_{2} . \\
& K(-2 O N)=\frac{1}{2 l-1} \sqrt{\frac{(l-m)(l-1-m)(\ell+m)(\ell-1+m)}{(2 l-3)(2 \ell+1)}} \times\left(\delta_{N O}-\delta_{N 2}\right) F_{O} \text {, } \\
& K(-2 I N)=\frac{1}{2(2 l-1)} \sqrt{\frac{(l-m)(l-1-m)(l-2-m)(l+m)}{(2 l-3)(2 l+1)}} \times\left(\delta_{N O}-\delta_{N 2}\right) P_{1} \text {, } \\
& K(-22 \mathrm{~N})=\frac{1}{4(2 l-1)} \sqrt{\frac{(l-\mathrm{m})(l-1-\mathrm{m})(l-2-\mathrm{m})(l-3-\mathrm{m})}{(2 l-3)(2 l+1)}} \times\left(\delta_{\mathrm{NO}}-\delta_{\mathrm{N} 2}\right) \mathrm{P}_{2} . \\
& \mathrm{K}(\text { ION })=\sqrt{\frac{(l+1-\mathrm{m})(\ell+1+\mathrm{m})}{\rho(2 \ell+1)(2 \ell+3)}}\left\{\left[\left(l+\mathrm{n}+\frac{3}{2}\right) \delta_{\mathrm{NO}}-\mathrm{n} \delta_{-\mathrm{N} I}\right]\right. \\
& \times\left[\frac{\partial}{\partial x_{3}}+\frac{1}{2}(l+2) \operatorname{div} \hat{u}_{3}-i m R_{0}\right]+\left[\left(l+n+\frac{3}{2}\right) \delta_{N O}\right. \\
& \left.+n \delta_{-N I}\right] \rho A_{3}-\left[\left(l+n+\frac{3}{2}\right)\left(l+n+\frac{5}{2}\right) \delta_{N I}-\left(n-\frac{3}{2}\right)\right. \\
& \left.\left.\times\left(l+n+\frac{3}{2}\right) \delta_{\text {NO }}-\mathrm{n}(l+\mathrm{n}+3) \delta_{-\mathrm{N} 1}+\mathrm{n}(\mathrm{n}-1) \delta_{-\mathrm{N} 2}\right] \mathrm{H}_{3}\right\}, \\
& K(I I N) \cdot=-\frac{1}{2} \sqrt{\frac{(l+1+m)(l+2+m)}{f(2 \ell+1)(2 \ell+3)}}\left\{\left[\left(l+n+\frac{3}{2}\right) \delta_{N O}-n \delta_{-N I}\right]\right. \\
& x\left[\frac{\partial}{\partial x_{1}}+i \frac{\partial}{\partial x_{2}}+\frac{1}{2}(l+2) \operatorname{div}\left(\hat{u}_{1}+i \hat{u}_{2}\right)-i\left(m-\frac{1}{2} \ell\right) R_{1}\right] \\
& +\left[\left(l+n+\frac{3}{2}\right) 8_{N O}+n \delta_{-N 1}\right] \rho\left(A_{1}+i A_{2}\right)-\left[\left(l+n+\frac{3}{2}\right)\right. \\
& \times\left(l+\mathrm{n}+\frac{5}{2}\right) \delta_{\mathrm{N} l}-\left(\mathrm{n}-\frac{3}{2}\right)\left(\ell+\mathrm{n}+\frac{3}{2}\right) \delta_{\mathrm{NO}}-\mathrm{n}(\ell+\mathrm{n}+3) \delta_{-\mathrm{Nl}}+
\end{aligned}
$$




$$
\begin{aligned}
& \left.\left.+n(n-I) \delta_{-N 2}\right]\left(H_{1}+i H_{2}\right)\right\}, \\
& \mathrm{K}(\mathrm{I2N})=\frac{1}{4} i \sqrt{\frac{(\ell-\mathrm{m})(\ell+\mathrm{I}+\mathrm{m})(\ell+2+\mathrm{m})(\ell+3+\mathrm{m})}{\rho(2 \ell+1)(2 \ell+3)}} \times\left[\left(\ell+\mathrm{n}+\frac{3}{2}\right) \delta_{\mathrm{NO}}-\mathrm{n} \delta_{-\mathrm{NI}}\right] \mathrm{R}_{2} . \\
& K(-I O N)=\sqrt{\frac{(l-m)(l+m)}{\rho(2 l-1)(2 l+1)}}\left\{\left(\delta_{\mathrm{NO}}-\delta_{\mathrm{NI}}\right)\left[\frac{\partial}{\partial x_{3}}-\frac{1}{2}(\ell-1) \operatorname{div} \hat{u}_{3}-\text { im } R_{\mathrm{O}}\right]\right. \\
& -\left(\delta_{\mathrm{NO}}+\delta_{\mathrm{N} 1}\right) \rho \mathrm{A}_{3}+\left[\left(l+\mathrm{n}+\frac{3}{2}\right) \delta_{\mathrm{N} 2}-(l+\mathrm{n}-1) \delta_{\mathrm{N} 1}\right. \\
& \left.\left.-\left(\mathrm{n}+\frac{5}{2}\right) \delta_{\mathrm{NO}}+\mathrm{n} \delta_{-\mathrm{NI}}\right] \mathrm{H}_{3}\right\}, \\
& K(-11 N)=\frac{1}{2} \sqrt{\frac{(l-1-m)(\ell-m)}{\rho(2 l-1)(2 l+1)}}\left\{( \delta _ { \mathrm { NO } } - \delta _ { \mathrm { N } 1 } ) \left[\frac{\partial}{\partial \mathrm{v}_{1}}+i \frac{\partial}{\partial x_{2}}\right.\right. \\
& \left.-\frac{1}{2}(\ell-1) \operatorname{div}\left(\hat{u}_{1}+i \hat{u}_{2}\right)-i\left(m+\frac{1}{2} \ell+\frac{1}{2}\right) R_{1}\right] \\
& -\left(\delta_{\mathrm{NO}}+\delta_{\mathrm{NI}}\right) \rho\left(\mathrm{A}_{1}+i \mathrm{~A}_{2}\right)+\left[\left(l+\mathrm{n}+\frac{3}{2}\right) \delta_{\mathrm{N} 2}\right. \\
& \left.\left.-(\ell+\mathrm{n}-1) \delta_{\mathrm{N} 1}-\left(\mathrm{n}+\frac{5}{2}\right) \delta_{\mathrm{NO}}+\mathrm{n} \delta_{-\mathrm{N} I}\right]\left(\mathrm{H}_{1}+\mathrm{iH}_{2}\right)\right\}, \\
& K(-12 N)=-\frac{1}{4} i \sqrt{\frac{(l-2-m)(\ell-I-m)(\ell-m)(\ell+I+m)}{\rho(2 l-1)(2 \ell+1)}} \times\left(\delta_{N O}-\delta_{N I}\right) R_{2} .
\end{aligned}
$$


THIS PAGE

\section{WAS INTENTIONALLY \\ LEFT BLANK}


INTERNAL DISTRIBUTION

ORNL/TM-5316

1-3. Lab Records

4. Lab Records - RC

5. Tech. Support Div., ORO, ERDA

6. Y-12 Document Reference

7-8. Central Research Library

9. Thermonuclear Library

10. ORNL Patent Office

11. J. D. Callen

12. J. F. Clarke

13. R. A. Dory

14. G. G. Kelley

15. D. G. McAlees

16. 0. B. Morgan

17. M. W. Rosenthal

18-43. W. I. van Rij

EXI'EKNAL DIS'TRIBUTION

44. Plasma Physics Library, Plasma Physics Laboratory, Princeton Univ., Forrestal Campus, P. O. Box 451, Princeton, NJ 08540

45. Controlled Thermonuclear Research Library, Lawrence Li.vermore Laboratory, P. O. Box 808, Livermore, CA 94550

46. Q Division Library, c/o F. L. Ribe, Los Alamos Scientific Laboratory, P. 0. Box 1663, Los Alamos, NM 87544

47. Controlled Thermonuclear Research Library, c/o Weston M. Stacey, Jr., Argonne National Laboratory, 9700 S. Cass Ave., Argonne, IL 60439

48. CTR Computer Center, c/o Dr. John Killeen, Lawrence Livermore Laboratory, P. O. Box 808, Livermore, CA 94550

49. Librarian, Culham Laboratory, U.K. Atomic Energy Authority, Abingdon, Oxon, OXI4, 3DB, United Kingdom

50. Ruth Lengye, Bibliothek, Max Planck Institut fur Plasmaphysik', 8046 Garching bei Munchen, Federal Republic of Germany

51. Library, Centre de Recherches en Physique des Plasma, 21 Avenue des Bains, 1007, Lausanne, Switzerland

52. A. M. Dupas, Documentation S.I.G.N., Department de La Physique du Plasma, Et de La Fusion Controlee, Association EURATOM-CEA, Sur La Fusion, Centre d'Etudes Nucleaires, BP 85 Centre Du TRI 38041 Grenoble Cedex (France)

53. Bibliothéque, Service du Confinement des Plasmas, C.E.A., B.P. No. 6, 92, Fontenay-aux-Roses (Seine)-France 
54. Library, International Centre for Theoretical Physics, Trieste, Italy

55. Library, Laboratorio Gas Ionizati, Frascatti; Italy

56. V. E. Ivanov, Physical-Technical Institute of the Ukranian Academy of Sciences, Sukhumi, U.S.S.R.

57. M. S. Rabinovich, Lebedev Inst1tute of Physics, Academy of Sc1ences of the U.S.S.R., Leninsky Prospect 53, Moscow, U.S.S.R.

58. Thermonuclear Laboratory, Kurchatov Institute of Atomic Energy, 46. Ulitsa Kurchatova, P. O. Box 3402, Moscow, U.S.S.R.

59. Library, Institute for Plasma Physics, Nagoya University, Nagoya, Japan 464

60. Libraryy, FOM-Instilut voor Flasma-Fysica, Rijnhuizen, Tutphaas, Netherlands

6.1. Plasma Physics Group, Department of Engineering Physics, Australian National University, P.O. Box 4, Canberra A.C.T. 2600 Australia

62. Thermonuclear Library, Japan Atomic Energy Research Institute, Tokai, Naka, Ibaraki, Japan

63. CTR Reading Room, c/o Prof. Dieter J. Sigmar, Room 37-391 M.I.T., Cambridge, MA 02139

64. CTR Reading Room, c/o Prof. D. W. Kerst, Dept. of Physics, Sterling IIäl, Univ. of Wioconein, Madisnn, WT 53706

65. CTR Reading Room; c/o Prof. I. B. Bernstein, Yale University, New Haven, CT 06510

66. Center for Plasma Physics and Thermonuclear Research, Univ. of Texas, Physics Building 330, Austin, TX 78712

67. CTR Reading Room, c/o Prot. B. D. Frled, Physics Dept., Univ. of California, Los Angeles, CA 90024

68. CTR Redine Ronm, c/o Prof, David C. Montegomery, Physics \& Astronomy Dept., Univ. of Iowa, Iowa City, IA 52240

69. Magneto-Fluid-Dynamics Library, Courant Inst. of Math. Sci., New York Univ., 251 Mercer St., New York, NY 10012

70. CTR Reading Room, c/o Prof. Allan N. Kaufman, Physics Dept., Univ. of California, Berkeley, CA 94720

71. CTR Reading Room, c/o Prof. W. B. Thompson, Univ. of California, San Diego, Physics Dept., La Jolla, CA 92037 
72. CTR Reading Room, c/o Prof. Alvin W. Trivelpiece, Dept. of

Physics \& Astronomy, Univ. of Maryland, College Park, MD 20742

73. CTR Reading Room, c/o Prof. T. Kammash, 103 Research Admin. Bldg., N. Campus, Univ. of Michigan, Ann Arbor, MI 48105

74. CTR Reading Room, c/o Dr. Ravi N. Sudan, Phillips Hall, Cornell Univ., Ithaca, NY 18450

75. Prof. Marshall N. Rosenbluth, Institute for Advanced Study, Princeton, NJ 08540

76. CTR Reading Room, c/o Prof. R. Gross, Plasma Research Lab., Columbia Univ., New York, NY 10027

77. CTR Reading Room, c/o Prof. Roy Gould, California Inst. of Tech., Pasadena, CA 91103.

78. Dr. Nicholas A. Krall, Science Applications, Inc., P.0. Box 2354,

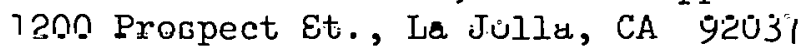

79. CTR Reading Room, c/o Dr. Jay P. Boris, Plasma Physics, Naval Research Laboratory, Washington, DC 20390

80. CTR Library, General Atomic Co., P.0. Box 81608, San Diego, CA 92138

81. CTR Library, c/o Dr. Alan F. Haught, United Technologies Research Labs, East Hartford, CT 06108

82. Dr. Robert ז. Price, Division of Controlled Thermonuclear Research, U. S. Energy Research and Development Administration, Washington, DC 20545

83. Dr. Bennett Miller, Division of Controlled Thermonuclear Research, U. S. Energy Research and Development Administration, Washington, DC 20545

84. Dr. Arthur Sleeper, Division of Controlled Thermonuclear Research, U. S. Energy Research and Development Administration, Washington, DC 20545

85. Dr. Walter Sadowski, Division of Controlled Thermonuclear Research, U. S. Energy Research and Development Administration, Washington, DC 20545

86. Dr. D. H. Priester, Division of Controlled Thermonuclear Research, U. S. Energy Research and Development Administration, Washington, DC 20545

87. Dr. Paul. H. Rutherford, Princeton Plasma Physics taab., Princeton Univ., Princeton, NJ 08540 
88. Dr. L. D. Pearlstein, L-388, Lawrence Livermore Laboratory, P.0. Box 808, Livermore, CA 94550

89. Dr. J. P. Friedberg, Los Alamos Scientific Laboratory, Los Alamos NM 87544

90. Dr. David W. Ross, Center for Plasma Physics \& Thermonuclear Research, Dept. of Physics, Univ. of Texas, Austin, TX 78712

91. Dr. Gareth E. Guest, General Atomic Co., P.0. Box 81608, San Diego, CA 92138

92. Dr. Claude Mercier, Service du Theorie des Plasmas, Centre d'Etudes Nucleaires, Fontenay-aux-Roses (Seine) France

93. Dr. J. B. Taylor, Culham Taboratory, UKAEA Ab1ngdun, Ox,on, OXI 3DB, United Kingdom

94. Dr. D. Pfirsch, Institute for Plasma Physics, 8046 Garching bei Munchen, Federa, Republic of Germany

95. Dr. V. D. Shafranov, I. V. Kurchatov Inst. of Atomic Energy, 46 Ulitsa Kurchatova, P.0. Box 3402 , Moscow, U.S.S.R.

96. Dr. Harold Grad, Courant Inst. of Math. Sci., New York Univ., 251 Mercer St., New York, NY 10012

97. Dr. J. G. Cordey, Culham Laboratory, UKAEA, Abingdon, Oxon, OXI4, 3DB, United Kingdom

98. Dr. David Baldwin, L-388, Lawrence Livermore Lab., P.0. Box 808, Ilverinure, CA 94550

99. Prof. Bruno Coppi, Dept. of Physics, M.I.T., Cambridge, MA 02139

100. Dr. Harold P. Furth, Princeton Hlasmá Physles Läi., Princcton Univ., P.0. Box 451, Princeton, NJ 08540

101-127. Tech. Information Center, E'RDA 